\title{
Cinética da dissolução redutiva da goethita e hematita em solos poligenéticos
}

\author{
Reductive dissolution kinetics of goethite and hematite in poligenetic soils
}

\author{
Alberto Vasconcellos Inda Junior ${ }^{1}$ Nestor Kämpf ${ }^{2}$ \\ Élvio Giasson ${ }^{2}$ Jacques Aveline Loureiro da Silva ${ }^{3}$
}

\section{RESUMO}

A cinética da dissolução redutiva da goethita e da hematita em solos poligenéticos foi avaliada por dois modelos matemáticos. O modelo Kabai descreveu a dissolução em um único segmento linear, não discriminando fases de um mesmo óxido ou de diferentes óxidos em cada amostra. A análise comparativa da estabilidade de cristais por este modelo somente pode ser realizada entre amostras distintas. O modelo Avrami-Erofejev descreveu a dissolução em mais de um segmento linear na maioria das amostras. Este modelo discriminou fases de um mesmo óxido e ofereceu condições para classificar o grau de estabilidade destes minerais dentro de cada amostra. Os modelos indicaram menor estabilidade da hematita em relação à goethita frente à dissolução redutiva, principalmente quando a última combinou baixa substituição por $\mathrm{Al}^{3+} \mathrm{e}$ alta área superficial específica. A influência destes parâmetros mineralógicos na estabilidade dos cristais à dissolução redutiva mostrou ser dependente da abordagem matemática utilizada para determinar a cinética dissolutiva.

Palavras-chave: óxidos de ferro, estabilidade diferencial, cristalinidade, substituição por $\mathrm{Al}^{3+}$.

\section{ABSTRACT}

Two mathematical models were used to evaluate the reductive dissolution kinetics of goethite and hematite. The Kabai's model described dissolution as a single linear segment, not discriminating the dissolution of different phases of the same iron oxide or different iron oxides in a sample. Consequently, this model can only compare crystal stability between distinct samples. The Avrami-Erofejev's model described dissolution as a set of linear segments for most of the samples. This model discriminated different phases of the same iron oxide and allowed classification of the degree of stability of these minerals in each sample. Both models indicated that hematite is less stable than goethite against reductive dissolution, mainly when goethite combines low $\mathrm{Al}^{+3}$ substitution and high specific surface area. The evaluation of these mineralogical parameters on the crystal stability in the reductive dissolution showed dependecy of the model used for determining the dissolutive kinetic.

Key words: iron oxides, differential stability, crystallinity, $\mathrm{Al}^{3+}$-substitution.

\section{INTRODUÇÃO}

Solos poligenéticos têm sua longa pedogênese freqüentemente associada a diferentes circunstâncias climáticas, geomorfológicas, etc., que certamente implicam na história complexa desses solos (MUGGLER, 1998). Como conseqüência, nestes solos, podem ocorrer diferentes gerações de óxidos de ferro como a goethita e/ou a hematita constituindo populações heterogêneas, nas quais os cristais possuem diferentes estabilidades a processos dissolutivos (INDA JUNIOR, 2002). A dissolução da goethita e hematita no solo é importante, pois pode alterar o comportamento químico e físico do solo e feições morfológicas como a sua cor. Em adição, a

\footnotetext{
${ }^{1}$ Engenheiro Agrônomo, Doutor, Professor do Departamento de Solos, Faculdade de Agronomia (FA), Universidade Federal do Rio Grande do Sul (UFRGS). CP 15100, 90001-970. Porto Alegre, RS, Brasil. E-mail: alberto.inda@ufrgs.br

${ }^{2}$ Engenheiro Agrônomo, PhD, Professor do Departamento de Solos, FA, UFRGS, Brasil.

${ }^{3}$ Bacharel em Matemática, PhD, Professor de Matemática Pura e Aplicada, Instituto de Matemática (IM), UFRGS, Brasil.
} 
dissolução desses minerais afeta a disponibilidade de nutrientes e a migração de poluentes em solos.

Considerável atenção tem sido dada à

dissolução ácida dos óxidos de ferro (SCHWERTMANN \& LATHAM, 1986; CORNELL \& GIOVANOLI, 1993; RUAN \& GILKES, 1995; WELLS et al., 2001) comparativamente à dissolução redutiva (TORRENT et al., 1987; JEANROY et al., 1991), embora a expressão desta última no ambiente pedogênico seja maior (SCHWERTMANN, 1991). Essas reações envolvem etapas que iniciam com a formação de complexos nos sítios superficiais, passando pelo enfraquecimento das ligações Fe-O e culminando no deslocamento do ferro para a solução. Entretanto, os mecanismos envolvidos em cada reação diferem. Ao contrário da reação ácida, na reação redutiva, ocorre um desbalanço de carga, devido à redução do $\mathrm{Fe}^{3+} \mathrm{a}$ $\mathrm{Fe}^{2+}$, que causa um desequilíbrio estrutural do cristal (SCHWERTMANN, 1991).

A etapa determinante da cinética dissolutiva é a reação de superfície, pois envolve maior energia de ativação. Neste sentido, a cristalinidade dos óxidos de ferro, expressa pelo domínio coerente médio (DCM) e área superficial específica (ASE) dos cristais, é determinante da estabilidade destes minerais à dissolução. A substituição isomórfica do $\mathrm{Fe}^{3+}$ por $\mathrm{Al}^{3+}$ na estrutura além de afetar a cristalinidade, pois causa uma contração na cela unitária devido o menor raio iônico do $\mathrm{Al}^{3+}(0,054 \mathrm{~nm})$ em relação ao $\mathrm{Fe}^{3+}(0,065$ $\mathrm{nm})$, determina uma maior estabilidade dos cristais à reações redutivas, devido ao estado de oxidação constante do alumínio.

Freqüentemente, os dados da dissolução são descritos por modelos matemáticos que fazem o ajuste linear dos dados experimentais, o que permite avaliar além da cinética dissolutiva, o efeito de parâmetros mineralógicos na estabilidade dos cristais (SCHWERTMANN, 1984; SINGH \& GILKES, 1992). Entretanto, não há concordância a respeito do modelo mais apropriado para descrever a dissolução dos óxidos de ferro. Dissoluções de misturas de goethita e hematita em amostras de solo indicam que a última dissolve preferencialmente, possibilitando a discriminação desses óxidos no solo (SCHWERTMANN \& LATHAM, 1986). Porém, em algumas situações, os cristais de goethita e hematita apresentam estabilidade similar, possivelmente devido a semelhanças na cristalinidade e na substituição por $\mathrm{Al}^{3+}$ destes minerais (TORRENT et al., 1987; SINGH \& GILKES, 1992). Resultados conflitantes refletem a expressiva amplitude das características mineralógicas da goethita e da hematita tanto entre diferentes solos (SCHWERTMANN \& KÄMPF, 1985) como em um mesmo solo, resultado da função profundidade do solo ou de alternâncias pedoambientais ao longo do desenvolvimento deste (poligênese) (MUGGLER, 1998; INDA JUNIOR, 2002).

Este estudo objetivou descrever a cinética da dissolução redutiva de goethitas e hematitas de solos poligenéticos através de dois modelos matemáticos; discriminar, nas amostras, a presença de cristais de um mesmo tipo (goethita ou hematita) com estabilidades distintas; verificar a estabilidade diferencial de goethitas e hematitas e a influência da cristalinidade e da substituição por $\mathrm{Al}^{3+}$ na estabilidade dos cristais.

\section{MATERIAL E MÉTODOS}

Foram selecionadas amostras da fração argila de 21 horizontes B e uma de saprolito de solos de diversas regiões do Brasil (Tabela 1), sendo onze amostras goethíticas e onze hematíticas. O ferro dos óxidos de ferro pedogênicos $\left(\mathrm{Fe}_{\mathrm{d}}\right)$ foi extraído a $80^{\circ} \mathrm{C}$, empregando-se solução de ditionito-citratobicarbonato de sódio em quatro extrações sucessivas (MEHRA \& JACKSON, 1960; INDA JUNIOR \& KÄMPF, 2003). O ferro dos óxidos de ferro de baixa cristalinidade $\left(\mathrm{Fe}_{\mathrm{o}}\right)$ foi extraído em solução de oxalato de amônio $0,2 \mathrm{~mol} \mathrm{~L}^{-1}$ à $\mathrm{pH} 3$, no escuro (SCHWERTMANN, 1964).

A difratometria de raios-X (DRX) foi realizada em difratômetro Philips, equipado com tubo de Co e filtro de Fe, em voltagem de $35 \mathrm{kV}$ e corrente de $25 \mathrm{~mA}$. As lâminas foram preenchidas com fração argila total não orientada. A não utilização da fração ferro concentrada objetivou evitar danos na superfície dos cristais pelo tratamento com $\mathrm{NaOH} 5 \mathrm{~mol} \mathrm{~L}^{-1}$ a quente. A varredura foi realizada no intervalo de 23 a $44{ }^{\circ} 2 \theta$, com um espaçamento entre leituras de $0,05{ }^{\circ} 2 \theta$ e tempo de leitura em cada passo de 10 segundos. A substituição por $\mathrm{Al}^{3+}$ na goethita foi estimada pela regressão $\mathrm{Al}\left(\mathrm{mol} \quad \mathrm{mol}^{-1}\right)=17,30-5,72 \mathrm{c}(\mathrm{nm})$ (SCHULZE, 1984); e na hematita pela regressão $\mathrm{Al}(\mathrm{mol}$ $\left.\mathrm{mol}^{-1}\right)=31,09-6,17 a_{0}(\mathrm{~nm})$ (SCHWERTMANN et al., 1979). A posição dos reflexos foi corrigida pelo reflexo da halita (padrão interno) e o desvio instrumental com amostra de quartzo ( $\varnothing$ entre $0,5-1,0 \mathrm{~mm})$. A cristalinidade foi avaliada através do domínio coerente médio dos cristais (DCM) perpendicular aos planos hkl 110 e 111 da goethita e 012, 104 e 110 da hematita (SCHULZE \& SCHWERTMANN, 1984). Aárea superficial específica da goethita foi estimada através da fórmula $A S E_{G t}=\left(1049 / D M C_{100}\right)-5 \quad\left(m^{2} \quad g^{-1}\right), \quad$ onde: $D M C_{100}=0,42 \quad D M C_{1} n m \quad$ (SCHULZE \& SCHWERTMANN, 1984), e da hematita através da 
Tabela 1 - Localização e classificação dos solos coletados, características da fração argila e dos óxidos de ferro.

\begin{tabular}{|c|c|c|c|c|c|c|c|c|c|c|c|c|}
\hline \multirow{3}{*}{ Amostra } & \multirow{3}{*}{ Local } & \multirow{3}{*}{ Classe $^{1}$} & \multirow{2}{*}{$\mathrm{Fe}_{\mathrm{d}}^{2}$} & \multirow{2}{*}{$\mathrm{Fe}_{\mathrm{o}}{ }^{3}$} & \multirow{3}{*}{$\mathrm{Fe}_{\mathrm{o}} / \mathrm{Fe}_{\mathrm{d}}$} & \multirow{4}{*}{$\begin{array}{c}\mathrm{Hm} / \\
(\mathrm{Hm}+\mathrm{Gt}) \\
0,00\end{array}$} & \multicolumn{6}{|c|}{ Goethita } \\
\hline & & & & & & & \multicolumn{3}{|c|}{$\mathrm{DCM}^{4}(\mathrm{~nm})$} & \multirow{2}{*}{$\begin{array}{c}\mathrm{I}^{5} \\
111 / 110\end{array}$} & \multirow{2}{*}{$\begin{array}{l}\mathrm{ASE}^{6} \\
\mathrm{~m}^{2} \mathrm{~g}^{-1}\end{array}$} & \multirow{2}{*}{$\begin{array}{c}\mathrm{Al}^{3+7} \\
\mathrm{~mol} \mathrm{~mol}^{-1}\end{array}$} \\
\hline & & & \multicolumn{2}{|c|}{$\mathrm{g} \mathrm{kg}^{-1}$} & & & 110 & \multicolumn{2}{|r|}{111} & & & \\
\hline G1 & SP & LA & 45,4 & 0,6 & 0,01 & & 35 & \multicolumn{2}{|r|}{-} & - & 66 & 0,35 \\
\hline G2 & $\mathrm{RJ}$ & LA & 48,3 & 0,4 & 0,01 & 0,00 & 27 & \multicolumn{2}{|r|}{ - } & - & 88 & 0,32 \\
\hline G3 & MG & $\mathrm{FX}$ & 110,6 & 0,5 & 0,00 & 0,00 & 35 & \multicolumn{2}{|r|}{62} & 1,77 & 66 & 0,18 \\
\hline G4 & $\mathrm{SP}$ & LA & 77,0 & 0,5 & 0,01 & 0,00 & 50 & \multicolumn{2}{|r|}{-} & - & 45 & 0,37 \\
\hline G5 & GO & LB & 77,0 & 0,5 & 0,01 & 0,21 & 26 & \multicolumn{2}{|r|}{-} & - & 91 & 0,26 \\
\hline G6 & SP & LA & 77,7 & 3,6 & 0,05 & 0,00 & 33 & \multicolumn{2}{|r|}{34} & 1,03 & 71 & 0,19 \\
\hline G7 & RS & SA & 199,5 & 2,1 & 0,01 & 0,00 & 21 & \multicolumn{2}{|r|}{30} & 1,43 & 114 & 0,01 \\
\hline G8 & MG & LB & 114,1 & 0,8 & 0,01 & 0,00 & 33 & \multicolumn{2}{|r|}{-} & - & 71 & 0,36 \\
\hline G9 & PR & LB & 79,1 & 1,1 & 0,01 & 0,00 & 15 & \multicolumn{2}{|r|}{24} & 1,60 & 162 & 0,25 \\
\hline G10 & RS & $\mathrm{CH}$ & 224,8 & 5,6 & 0,02 & 0,00 & 23 & \multicolumn{2}{|r|}{33} & 1,43 & 104 & 0,09 \\
\hline G11 & MG & LA & 54,0 & 0,8 & 0,01 & 0,00 & 30 & \multicolumn{2}{|r|}{-} & - & 78 & 0,35 \\
\hline \multirow{3}{*}{ Amostra } & \multirow{3}{*}{ Local } & \multirow{3}{*}{ Classe } & & & & & & & & Hemati & & \\
\hline & & & $\mathrm{Fe}_{\mathrm{d}}$ & $\mathrm{Fe}_{\mathrm{o}}$ & $\mathrm{Fe}_{\mathrm{o}} / \mathrm{Fe}_{\mathrm{d}}$ & $\mathrm{Hm} /$ & & $\mathrm{M}(\mathrm{n}$ & m) & I & ASE & $\mathrm{Al}^{3+}$ \\
\hline & & & & & & & 012 & 110 & 104 & $110 / 104$ & $m^{2} g^{-1}$ & $\mathrm{~mol} \mathrm{~mol}^{-1}$ \\
\hline H12 & SP & $\mathrm{LV}$ & 141,5 & 2,9 & 0,02 & 0,90 & 32 & 31 & 22 & 1,41 & 55 & 0,12 \\
\hline H13 & PR & LV & 126,4 & 3,4 & 0,03 & 0,87 & - & 25 & 20 & 1,25 & - & 0,10 \\
\hline H14 & MG & LV & 267,6 & 4,8 & 0,02 & 0,91 & 37 & 47 & 28 & 1,68 & 40 & 0,09 \\
\hline H15 & $\mathrm{SP}$ & LV & 147,0 & 3,9 & 0,03 & 0,96 & - & 37 & 22 & 1,68 & - & 0,12 \\
\hline H16 & SP & LV & 150,7 & 3,1 & 0,02 & 0,97 & 37 & 49 & 25 & 1,96 & 39 & 0,12 \\
\hline H17 & RS & LV & 99,2 & 3,1 & 0,03 & 0,90 & 56 & - & 21 & - & - & 0,08 \\
\hline H18 & SP & $\mathrm{LV}$ & 129,2 & 4,2 & 0,03 & 0,96 & 33 & 32 & 30 & 1,07 & 53 & 0,13 \\
\hline H19 & SP & $\mathrm{LV}$ & 153,4 & 4,8 & 0,03 & 0,95 & 32 & 58 & 24 & 2,42 & 39 & 0,12 \\
\hline H20 & MG & LV & 157,2 & 5,2 & 0,03 & 0,95 & 31 & 38 & 22 & 1,73 & 49 & 0,14 \\
\hline H21 & PR & LV & 132,4 & 6,1 & 0,05 & 0,93 & 32 & 31 & 19 & 1,63 & 55 & 0,10 \\
\hline H22 & GO & LV & 106,1 & 4,4 & 0,04 & 0,94 & - & 33 & 23 & 1,43 & - & 0,12 \\
\hline
\end{tabular}

${ }^{1}$ Embrapa (1999) - LA= Latossolo Amarelo; FX= Plintossolo Háplico; LB= Latossolo Bruno; CH= Cambissolo Húmico;

$\mathrm{SA}=$ Saprolito; LV= Latossolo Vermelho ${ }^{2}$ Extraído com DCB ${ }^{3}$ Extraído com oxalato de amônio ${ }^{4}$ Domínio coerente médio dos cristais; ${ }^{5}$ Razão da intensidade dos reflexos ${ }^{6}$ Área superficial específica ${ }^{7}$ Substituição do $\mathrm{Fe}^{3+}$ por $\mathrm{Al}^{3+}$

fórmula $A S E_{H m}=2(r+h) 10^{3} / r h d\left(m^{2} g^{-1}\right)$, onde: $r=0,71 /$ $2\left(D M C_{110}\right) ; h=0,59 D M C_{012} ; \quad d=5,26 \mathrm{~g} / \mathrm{cm}^{3}$ (SCHWERTMANN \& KÄMPF, 1985). A razão Hm/ $(\mathrm{Hm}+\mathrm{Gt})$ foi calculada dos reflexos 012 da hematita e 110 da goethita.

A dissolução seletiva dos óxidos de ferro nas amostras foi realizada em onze etapas controladas pelo tempo (5, 10, 20, 40, 60, 120, 240, 480, 720, 1920 e 3840 minutos). A cada etapa de dissolução, uma alíquota de cada amostra, contendo $100 \mathrm{mg}$ de $\mathrm{Fe}_{\mathrm{d}}$, foi agitada $\left(50 \mathrm{rpm} ; 20^{\circ} \mathrm{C} \pm 2\right.$ ) em $40 \mathrm{~mL}$ de solução citrato-Na $0,3 \mathrm{~mol} \mathrm{~L}{ }^{-1}$ : bicarbonato-Na $1 \mathrm{~mol} \mathrm{~L}{ }^{-1}$, na razão 8:1; e $2 \mathrm{~g}$ de ditionito de sódio. Ao final de cada etapa, as amostras foram centrifugadas (3000rpm/5') e o extrato coletado foi completado a $50 \mathrm{~mL}$. Os teores de ferro e alumínio foram determinados por espectroscopia de absorção atômica.

\section{RESULTADOS E DISCUSSÃO}

Descrição mineralógica e cristalográfica das amostras

As características das amostras goethíticas e hematíticas constam na tabela 1 . As formas de ferro nas amostras apresentaram as seguintes amplitudes: 45 a $267 \mathrm{~g} \mathrm{~kg}^{-1}$ de $\mathrm{Fe}_{\mathrm{d}}$; e 0,4 a 6,1 $\mathrm{g} \mathrm{kg}^{-1}$ de $\mathrm{Fe}_{\mathrm{o}}$. Os baixos valores da razão $\mathrm{Fe}_{\mathrm{o}} / \mathrm{Fe}_{\mathrm{d}}(<0,05)$ indicaram a predominância de óxidos de ferro cristalinos. Exceto a amostra G5, com presença de hematita, e a G7, com presença de lepidocrocita, a goethita foi o único óxido de ferro identificado nas amostras goethíticas. Embora dominante nas amostras hematíticas, a hematita ocorreu sempre associada com goethita e, freqüentemente, com presença de maghemita.

Na goethita o $\mathrm{DCM}_{110}$ variou de 15 a $50 \mathrm{~nm}$, amplitude similar a de goethitas de solos tropicais 
(DICK, 1986). O DCM ${ }_{111}$ da goethita foi calculado somente em cinco amostras devido à interferência do reflexo 021 da gibbsita, tendo variado de 24 a 62nm. Em quatro delas (G3, G7, G9 e G10), que incluem as três amostras não latossólicas, a razão $\mathrm{DCM}_{111} / \mathrm{DCM}_{110}$ variou entre 1,43 e 1,77, sugerindo forma acicular. Na amostra G6 o $\mathrm{DCM}_{111}$ foi semelhante ao $\mathrm{DCM}_{110}$, indicando cristais isodimensionais (SCHWERTMANN \& KÄMPF, 1985). Na hematita, o DCM mostrou as seguintes amplitudes: 25 a 58nm para o $\mathrm{DCM}_{110}$; 19 a $30 \mathrm{~nm}$ para o $\mathrm{DCM}_{104}$; e 31 a $56 \mathrm{~nm}$ para o $\mathrm{DCM}_{012}$. Os valores da razão $\mathrm{DCM}_{110} / \mathrm{DCM}_{104}$, maiores que a unidade sugerem cristais em forma de placas (DICK, 1986). A ASE da goethita variou de 45 a $162 \mathrm{~m}^{2} \mathrm{~g}^{-1}$, enquanto, para a hematita, a amplitude foi menor (39 a $55 \mathrm{~m}^{2} \mathrm{~g}^{-1}$ ), muito embora não tenha sido possível estimar este parâmetro em todas as amostras devido à interferência do reflexo 002 da caulinita sobre o reflexo 012 da hematita.

A substituição por $\mathrm{Al}^{3+}$ na goethita variou de 0,01 a $0,38 \mathrm{~mol} \mathrm{~mol}^{-1}$. Nos difratogramas das amostras G1, G3, G6 e G8, o reflexo 111 apresentou-se duplicado, determinando a obtenção de espaçamentos $d_{111}$ mínimos e $d_{111}$ máximos, os quais refletiram, respectivamente, a presença goethitas com valores de substituição por $\mathrm{Al}^{3+}$ altos $\left(>0,22 \mathrm{~mol} \mathrm{~mol}^{-1}\right)$ e baixos $\left(0,14\right.$ a $\left.0,19 \mathrm{~mol} \mathrm{~mol}^{-1}\right)$ nessas amostras, confirmando registros da coexistência de cristais com diferentes graus de substituição por $\mathrm{Al}^{3+}$ em uma mesma população de goethita (MUGGLER, 1998). Nas hematitas, a substituição por $\mathrm{Al}^{3+}$ variou entre 0,08 e $0,14 \mathrm{~mol} \mathrm{~mol}^{-1}$.

Curvas da dissolução redutiva e congruência da dissolução de $\mathrm{Fe}$ e $\mathrm{Al}$

Após 3840 minutos, a dissolução da goethita foi total nas amostras não latossólicas (G3, G7 e G10) e variou entre 59 e 96\% nas latossólicas (Figura 1 a). A hematita dissolveu totalmente em 480 minutos nas amostras H18 e H22, e em 720 minutos nas demais (Figura $1 \mathrm{~b}$ ). As amostras goethíticas latossólicas e do Plintossolo apresentaram curvas desaceleratórias, evidenciadas pela diminuição da declividade das mesmas, indicando claramente mudanças na cinética dissolutiva da goethita. Nas amostras hematíticas e goethíticas não latossólicas (G7 e G10), a dissolução foi linear até aproximadamente 80\% da solubilização do ferro. Em dissoluções ácidas, a hematita apresentou comportamento similar, possivelmente devido ao ordenamento interno dos cristais ser equidirecional, fato que restringe as reações à superfície externa dos mesmos (CORNELL \& GIOVANOLI, 1993). Na goethita, a ocorrência de interdomínios estruturais determina que, em dissoluções ácidas, as curvas sejam sigmoídais, resultado de um fracionamento inicial dos cristais (aumenta a ASE) causado pela penetração de prótons nos interdomínios (SCHWERTMANN, 1984; RUAN \& GILKES, 1995). No presente estudo, as curvas desaceleratórias obtidas na dissolução redutiva podem ser resultado da não penetração dos transportadores de elétrons nos interdomínios da goethita, no caso o ânion ditionito $\left(\mathrm{S}_{2} \mathrm{O}_{4}{ }^{2-}\right.$ ) (segundo os raios de Van Der Waals mede $0,742 \mathrm{~nm}$ na maior dimensão), limitando assim a dissolução à superfície externa dos cristais (TORRENT et al., 1987), semelhante ao que ocorreu na dissolução da hematita.

A relação entre os teores de ferro e alumínio dissolvidos ao longo das dissoluções nas amostras goethíticas foi congruente (Figura $1 \mathrm{c}$ ), indicando que o alumínio estava uniformemente distribuído nos cristais de goethita. Para as amostras hematíticas, a tendência de distribuição côncava dos dados indicou uma tendência de maior concentração do alumínio em direção ao centro dos cristais de hematita (Figura $1 \mathrm{~d}$ ).

\section{Cinética da dissolução redutiva}

A dissolução redutiva da goethita e da hematita foi descrita por dois modelos matemáticos. No modelo Kabai $\left(C / C_{o}=1-e^{-(k t)} a\right)$ (KABAI, 1973), $C$ e $C_{o}$ representam a fração de ferro dissolvida no tempo $t$ e a fração de ferro no tempo $t=0$ (100mg de $\mathrm{Fe}_{\mathrm{d}}$ ), respectivamente, $k$ é a taxa constante de dissolução e $a$ é a constante de ordem média da reação. Para $a>1$, a curva de dissolução é sigmoidal e para $a<1$, a curva de dissolução é desaceleratória. A forma linear deste modelo é expressa como $\ln \ln (1 /(1-C)$ Co $)=a \operatorname{lnk}+a \operatorname{lnt}$. A relação $\ln \ln \left(1 /\left(1-C / C_{0}\right)\right)$ versus ln $t$ (tempo de dissolução) descreveu a dissolução da goethita e da hematita nas amostras em um único segmento linear (Figura $2 \mathrm{a}, \mathrm{b})$. A constante da ordem média da reação $(a)$, menor que a unidade em todas as amostras (Tabela 2), indicou que a dissolução procedeu da superfície para o centro dos cristais (KABAI, 1973). Entretanto, valores de a próximos da unidade (ex. G3, G7 e G10) sugerem que o mecanismo anterior pode ter atuado em combinação com a desintegração parcial de cristais.

$\mathrm{O}$ ajuste dos dados pelo modelo Kabai em somente um segmento linear não discriminou diferentes fases de goethita ou hematita nas amostras, mesmo considerando a provável coexistência de cristais de goethita com diferentes graus de substituição por $\mathrm{Al}^{3+}$ nas amostras G1, G3, G6 e G8; as associações goethitahematita e goethita-lepidocrocita, respectivamente, nas amostras G5 e G7; e pequenos conteúdos de goethita 
(a)

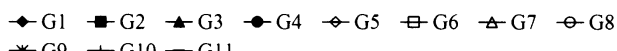

$$
\begin{aligned}
& \text { * G9+G10-G11 }
\end{aligned}
$$

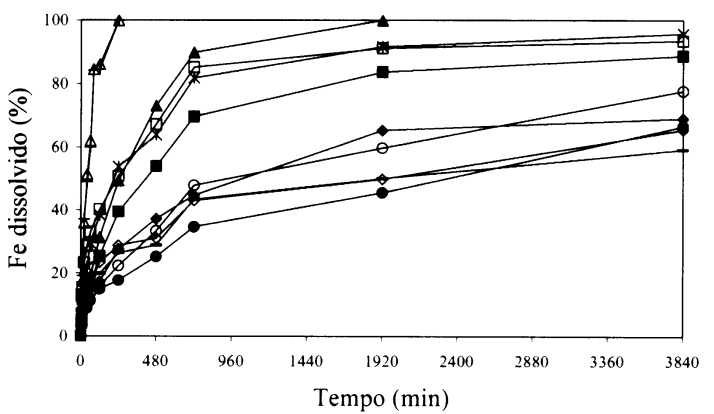

(c)

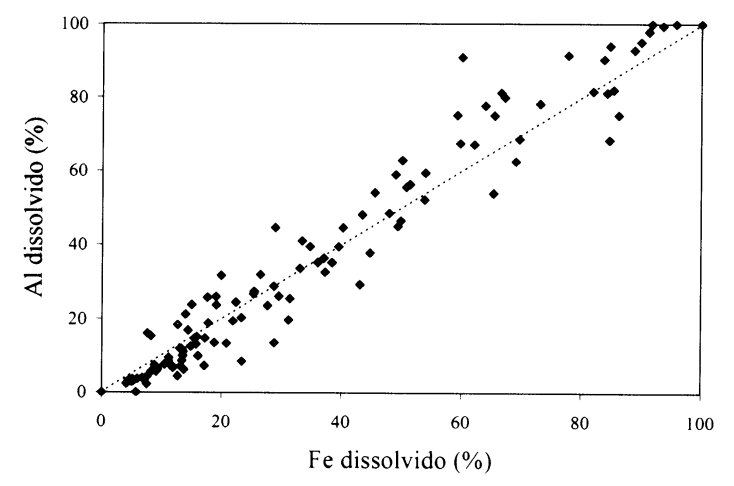

(b)

$\rightarrow \mathrm{H} 12 \rightarrow-\mathrm{H} 13 \rightarrow \mathrm{H} 14 \rightarrow \mathrm{H} 15 \rightarrow \mathrm{H} 16 \rightarrow \mathrm{H} 17 \rightarrow \mathrm{H} 18 \rightarrow-\mathrm{H} 19$ $* \mathrm{H} 20+\mathrm{H} 2 \mathrm{I}-\mathrm{H} 22$

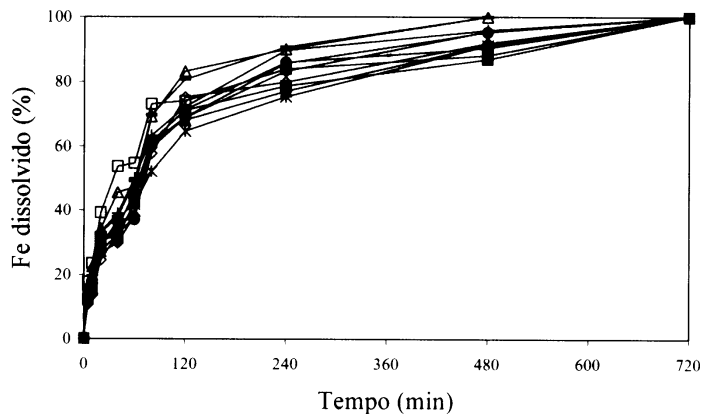

(d)

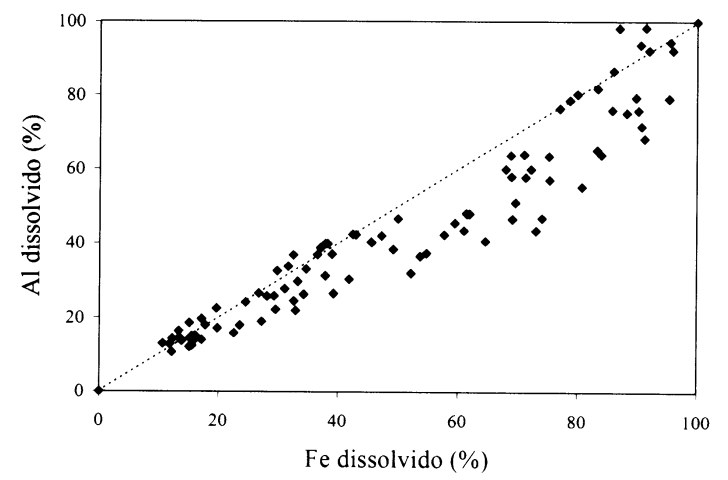

Figura 1 - Percentagem de ferro dissolvido ao longo da tempo de dissolução das amostras goethíticas (a) e hematíticas (b), e a relação entre a solubilização do ferro e do alumínio na dissolução das amostras goethíticas (c) e hematíticas (d).

nas amostras hematíticas (Tabela 1). Em razão disso, a taxa constante de dissolução $(k)$, derivada do segmento linear de cada amostra, foi interpretada como um indicativo da estabilidade média dos cristais contidos em uma mesma amostra. Esta concepção permitiu a análise comparativa somente entre os cristais (goethita e hematita) de diferentes amostras, a partir de parâmetros mineralógicos obtidos pela metodologia tradicional, cuja utilização também pressupõe os óxidos de ferro no solo como populações homogêneas.

As amostras goethíticas e hematíticas apresentaram, respectivamente, as seguintes amplitudes para valores de $k$ : 0,18 a 18,93; e 8,32 a 14,28 x $10^{3} \mathrm{~min}^{-1}$ (Tabela 2). Esses valores superam os obtidos por SCHWERTMANN \& LATHAM (1986) e SINGH \& GILKES (1995) em dissoluções ácidas, provavelmente devido à maior efetividade do mecanismo de dissolução redutiva, o qual envolve intenso desbalanço de carga na estrutura a partir da redução dos íons $\mathrm{Fe}^{3+}$. Nas amostras goethíticas latossólicas e do Plintossolo, 88 \% da variabilidade de $k$ foi explicada pela substituição por $\mathrm{Al}^{3+}$ na goethita (Tabela 1 ), com base na análise de regressão simples $k=17,58-53,05 \mathrm{Al}^{3+} ; \mathrm{n}=11$. Estes resultados confirmam em parte o efeito estabilizante do $\mathrm{Al}^{3+}$ em goethitas e hematitas sintéticas submetidas a dissoluções redutivas (TORRENT et al., 1987), uma vez que a mesma relação não foi observada para as amostras hematíticas, possivelmente devido à pequena variação na substituição por $\mathrm{Al}^{3+}$ entre as hematitas (Tabela 1). Valores de $k$ obtidos nas amostras de ambos os grupos não apresentaram relação significativa com os demais parâmetros mineralógicos (DMC $\mathrm{Dkl}_{\mathrm{hk}}$, ASE) estimados para a goethita e hematita. Nas amostras goethíticas, este fato pode ser conseqüência da heterogeneidade (não discriminada pelo modelo Kabai) dos cristais dentro das amostras; enquanto nas hematíticas decorre provavelmente da semelhança mineralógica entre os cristais de hematita nas amostras estudadas. 
(a)

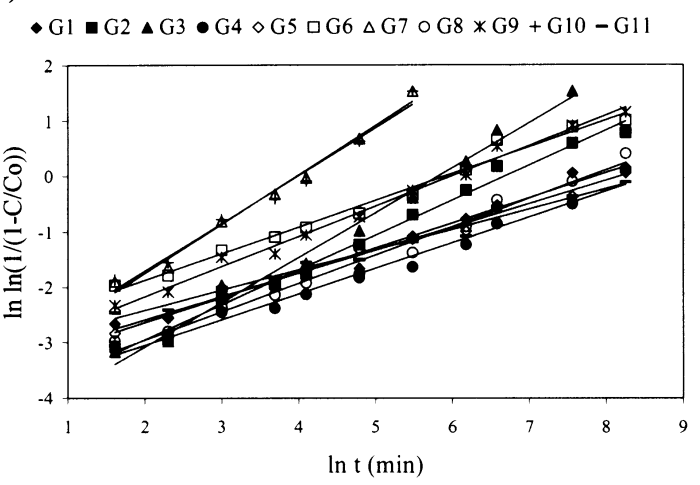

(c)

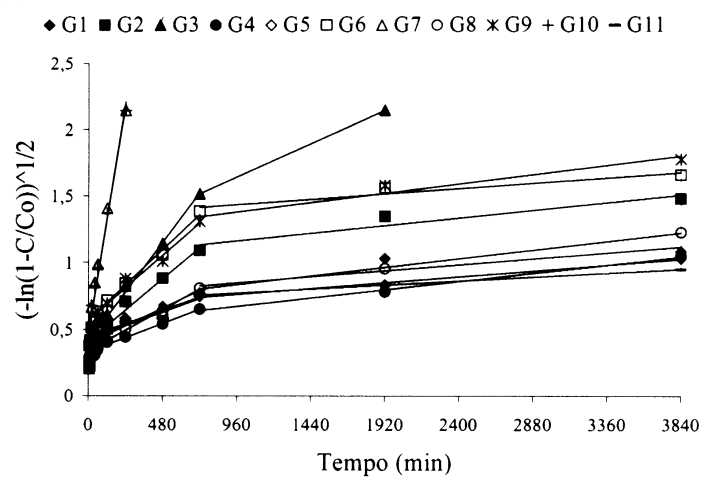

(b)

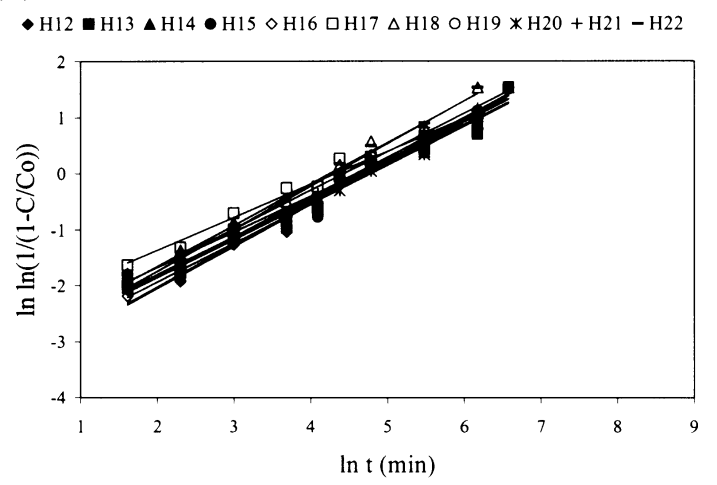

(d)

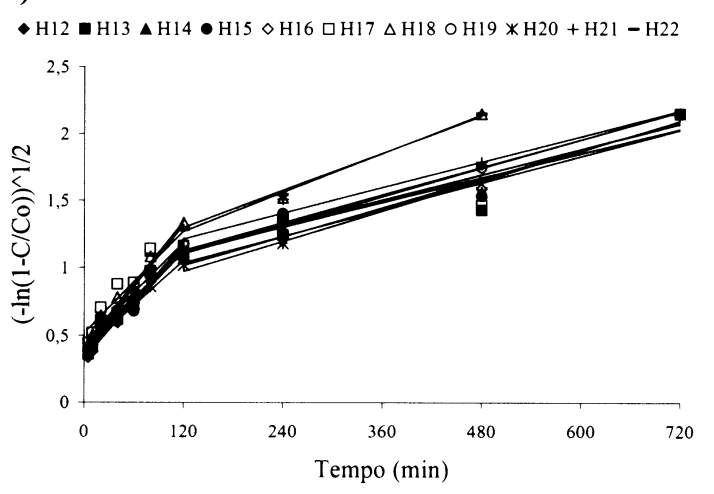

Figura 2 - Curvas de dissolução das amostras goethíticas (G) e hematíticas (H) descritas pelos modelos lineares Kabai (a, b) e AvramiErofejev (c, d).

Comparando o conjunto das amostras, a G7 e a G10, constituídas por goethitas de baixa substituição por $\mathrm{Al}^{3+}$ e alta ASE, apresentaram os maiores valores de $k$ (Tabela 2). Nas demais amostras goethíticas (latossólicas e plintossólica), o $k$ foi em média $\left(1,23 \times 10^{3} \mathrm{~min}^{-1}\right)$ nove vezes inferior ao da hematita nas amostras hematíticas $\left(10,69 \times 10^{3} \mathrm{~min}^{-1}\right)$, apesar da menor ASE estimada para as hematitas nestas últimas.

O modelo Avrami-Erofejev é expresso como $k t=[-\ln (1-C / C o)]^{1 / 2}(C O R N E L L \&$ GIOVANOLI, 1993), onde $C$ e $C_{o}$ representam a fração de ferro dissolvida no tempo $t$ e a fração de ferro no tempo $t=0(100 \mathrm{mg}$ de $\mathrm{Fe}_{\mathrm{d}}$ ), respectivamente, e $k$ é a taxa constante de dissolução. A relação $[-\ln (1-C / C o)]^{1 / 2}$ versus $t$ (tempo de dissolução) descreveu a dissolução da goethita em três segmentos lineares na maioria das amostras, com exceção da G7 e G10, descritas em um único segmento linear (Figura 2 c); e a dissolução da hematita em dois segmentos lineares em todas as amostras (Figura $2 \mathrm{~d}$ ). Estes resultados colaboram com a hipótese da coexistência de cristais de um mesmo tipo, porém com estabilidades distintas, resultado da poligênese desses solos (MUGGLER, 1998) e confirmam aqueles obtidos por WELLS et al. (2001) em dissoluções ácidas de hematitas sintéticas.

Os teores de ferro relativos aos cristais de goethita ou hematita dissolvidos nos diferentes segmentos lineares $(\% \mathrm{Fe} / \mathrm{SL})$ e os valores de $k$ derivados de cada segmento constam na tabela 2 . Com base nos valores de $k$, os cristais de goethita e hematita foram classificados quanto ao grau de estabilidade à dissolução redutiva conforme a seguinte escala: muito baixa $\left(k^{3} 5,6 \times 10^{-3} \mathrm{~min}^{-1}\right)$, baixa $\left(5,6>k^{3} 2,2 \times 10^{-3} \mathrm{~min}^{-1}\right)$, média $\left(2,2>k^{3} 0,4 \times 10^{-3} \mathrm{~min}^{-1}\right)$ e alta $\left(k<0,4 \times 10^{-3} \mathrm{~min}^{-1}\right)$.

Conforme esta escala, a totalidade dos cristais de goethita nas amostras G7 e G10 (não latossólicas), apresentaram uma estabilidade muito baixa a dissolução redutiva. A dissolução foi descrita em somente um segmento linear com valores médios de $k$ para as duas amostras de $7,25 \times 10^{-3} \mathrm{~min}^{-1}$. Estes resultados estão de acordo com os ambientes

Ciência Rural, v.35, n.3, mai-jun, 2005. 
Tabela 2 - Parâmetros da dissolução redutiva das amostras goethíticas (G) e hematíticas (H).

\begin{tabular}{|c|c|c|c|c|c|c|c|c|c|c|c|c|}
\hline \multirow{2}{*}{ Amostra } & \multicolumn{6}{|c|}{ Equação Kabai } & \multirow{2}{*}{ Amostra } & \multicolumn{5}{|c|}{ Equação Avrami-Erofejev } \\
\hline & $\mathrm{SL}^{1}$ & $\alpha^{2}$ & k. $10^{3} \min ^{-13}$ & $\% \mathrm{Fe} / \mathrm{SL}^{4}$ & $\mathrm{n}$ & $\mathrm{r}$ & & SL & $\mathrm{k} \cdot 10^{3} \min ^{-1}$ & $\% \mathrm{Fe} / \mathrm{SL}$ & $\mathrm{n}$ & $\mathrm{r}$ \\
\hline \multirow{4}{*}{ G1 } & \multirow{3}{*}{ I } & \multirow{4}{*}{0,450} & \multirow{3}{*}{0,39} & \multirow{3}{*}{69} & \multirow{3}{*}{11} & \multirow{3}{*}{0,991} & \multirow{3}{*}{ G1 } & I & 2,70 & 16 & 5 & 0,949 \\
\hline & & & & & & & & II & 0,50 & 46 & 5 & 0,981 \\
\hline & & & & & & & & III & 0,03 & 7 & 3 & 1 \\
\hline & \multirow{3}{*}{ I } & & \multirow{3}{*}{1,26} & & & & & I & 3,70 & 16 & 5 & 0,945 \\
\hline \multirow[t]{2}{*}{ G2 } & & \multirow[t]{2}{*}{0,630} & & 89 & 11 & 0,995 & G2 & II & 1,00 & 64 & 5 & 0,987 \\
\hline & & & & & & & & III & 0,07 & 9 & 3 & 1 \\
\hline & & & & & & & & I & 4,30 & 19 & 5 & 0,913 \\
\hline G3 & I & 0,809 & 3,01 & 100 & 10 & 0,992 & G3 & II & 1,50 & 71 & 5 & 0,997 \\
\hline & & & & & & & & III & 0,50 & 10 & 2 & 1 \\
\hline & & & & & & & & I & 2,20 & 11 & 5 & 0,941 \\
\hline G4 & I & 0,463 & 0,19 & 67 & 11 & 0,991 & G4 & II & 0,40 & 24 & 5 & 0,998 \\
\hline & & & & & & & & III & 0,10 & 32 & 3 & 0,997 \\
\hline & & & & & & & & I & 3,20 & 17 & 5 & 0,944 \\
\hline G5 & I & 0,418 & 0,28 & 65 & 11 & 0,994 & G5 & II & 0,40 & 26 & 5 & 0,960 \\
\hline & & & & & & & & III & 0,09 & 22 & 3 & 0,994 \\
\hline & & & & & & & & I & 4,60 & 33 & 5 & 0,960 \\
\hline G6 & I & 0,482 & 2,76 & 94 & 11 & 0,991 & G6 & II & 1,10 & 55 & 5 & 0,996 \\
\hline & & & & & & & & III & 0,05 & 6 & 3 & 1 \\
\hline G7 & I & 0,888 & 18,93 & 100 & 7 & 0,992 & G7 & I & 7,30 & 100 & 7 & 0,989 \\
\hline & & & & & & & & I & 2,70 & 14 & 5 & 0,966 \\
\hline G8 & I & 0,511 & 0,42 & 78 & 11 & 0,991 & G8 & II & 0,60 & 34 & 5 & 0,999 \\
\hline & & & & & & & & III & 0,10 & 30 & 3 & 0,999 \\
\hline & & & & & & & & I & 4,70 & 30 & 5 & 0,939 \\
\hline G9 & I & 0,545 & 2,53 & 96 & 11 & 0,995 & G9 & II & 1,00 & 52 & 5 & 0,987 \\
\hline & & & & & & & & III & 0,10 & 14 & 3 & 0,976 \\
\hline G10 & I & 0,861 & 18,63 & 100 & 7 & 0,990 & G10 & I & 7,20 & 100 & 7 & 0,992 \\
\hline & & & & & & & & I & 3,00 & 19 & 5 & 0,979 \\
\hline G11 & I & 0,365 & 0,18 & 59 & 11 & 0,991 & G11 & II & 0,40 & 24 & 5 & 0,969 \\
\hline & & & & & & & & III & 0,06 & 16 & 3 & 0,999 \\
\hline H12 & $\mathrm{I}$ & 0751 & 916 & 100 & 10 & ๑ 990 & H12 & I & 6,40 & 69 & 7 & 0,979 \\
\hline HIL & 1 & U,ל & 9,16 & 100 & 10 & 0,990 & H12 & II & 1,80 & 31 & 4 & 0,999 \\
\hline$H 12$ & $\mathrm{I}$ & 0691 & 809 & 100 & 10 & 0904 & $H 12$ & I & 6,40 & 71 & 7 & 0,975 \\
\hline H13 & 1 & 0,081 & 8,88 & 100 & 10 & 0,984 & H13 & II & 1,60 & 29 & 4 & 0,950 \\
\hline $\mathrm{H} 14$ & $\mathrm{I}$ & 0627 & 9 75 & 100 & 10 & 0907 & H14 & I & 5,70 & 68 & 7 & 0,977 \\
\hline H14 & 1 & 0,637 & 9,75 & 100 & 10 & 0,987 & H14 & II & 1,80 & 32 & 4 & 0,989 \\
\hline H15 & $\mathrm{I}$ & 0602 & 067 & 100 & 10 & 0902 & $H 15$ & I & 6,20 & 71 & 7 & 0,975 \\
\hline H15 & 1 & 0,083 & 9,02 & 100 & 10 & 0,983 & H15 & II & 1,60 & 29 & 4 & 0,965 \\
\hline$H 16$ & I & 0740 & 875 & 100 & 10 & 0990 & H16 & I & 7,10 & 75 & 7 & 0,991 \\
\hline П110 & 1 & 0,149 & 0,15 & 100 & 10 & 0,990 & Н10 & II & 1,60 & 25 & 4 & 0,981 \\
\hline $\mathrm{H} 17$ & $\mathrm{I}$ & 0.587 & 13.22 & 100 & 10 & 0984 & $\mathrm{H} 17$ & I & 6,30 & 74 & 7 & 0,937 \\
\hline & & & 13,22 & 100 & 10 & 0,504 & ח11 & II & 1,50 & 26 & 4 & 0,944 \\
\hline H18 & $\mathrm{J}$ & 0736 & 1428 & 100 & 9 & 0989 & H18 & I & 7,80 & 83 & 7 & 0,984 \\
\hline & & 0,130 & 14,28 & 100 & $y$ & 0,989 & Н18 & II & 2,30 & 17 & 3 & 0,993 \\
\hline H19 & I & 0.707 & 10.18 & 100 & 10 & 0995 & H19 & I & 7,70 & 69 & 7 & 0,986 \\
\hline HI9 & 1 & $0, \pi /$ & 10,18 & 100 & 10 & 0,995 & H19 & II & 1,70 & 31 & 4 & 0,999 \\
\hline $\mathrm{H} 2 \mathrm{O}$ & I & 0.694 & 8.32 & 100 & 10 & 0.991 & H20 & I & 5,60 & 65 & 7 & 0,984 \\
\hline & & & & & & & & II & 1,90 & 35 & 4 & 0,992 \\
\hline H21 & $\mathrm{I}$ & 0696 & 1181 & 100 & 10 & 0905 & H21 & I & 6,30 & 72 & 7 & 0,978 \\
\hline П 21 & 1 & , ,080 & 11,04 & 100 & 10 & 0,995 & Н 21 & II & 1,60 & 28 & 4 & 0,984 \\
\hline $\mathrm{H} 22$ & $\mathrm{I}$ & 0750 & 1250 & 100 & 0 & 0907 & רכרע & I & 7,70 & 81 & 7 & 0,986 \\
\hline H 22 & 1 & 0,159 & 13,59 & 100 & 9 & $0,98 /$ & H2L & II & 2,40 & 19 & 3 & 0,999 \\
\hline
\end{tabular}

${ }^{1}$ Segmento linear ${ }^{2}$ Constante de ordem média da reação ${ }^{3}$ Taxa constante de dissolução

${ }^{4}$ Ferro dissolvido em cada segmento linear

Ciência Rural, v.35, n.3, mai-jun, 2005. 
pedogênicos em estágio inicial (G7) e incipiente (G10) de formação, representados por goethitas homogêneas, as quais apresentaram baixa substituição por $\mathrm{Al}^{3+}$ e alta ASE (Tabela 1). Estabilidade muito baixa também foi apresentada, em média, por 73\% dos cristais de hematita nas amostras hematíticas, dissolvidos no primeiro segmento linear, com valores de $k$ que variaram entre 5,6 e 7,8 x 10-3 $\mathrm{min}^{-1}$ (média de 6,65 x 10-3 $\mathrm{min}^{-1}$ ). A similar estabilidade da hematita nas amostras latossólicas (Tabela 2), bem como a pequena amplitude de suas características (Tabela 1), está, possivelmente, relacionada à maior especificidade de seu ambiente de formação em relação à goethita (SCHWERTMANN \& TAYLOR, 1989).

Nas amostras goethíticas latossólicas e plintossólica, o primeiro segmento linear descreveu em média a dissolução de $20 \%$ dos cristais de goethita, cujos valores de $k$ entre 2,2 e 4,7 x 10 $0^{-3} \mathrm{~min}^{-1}$ (média de $3,46 \times 10^{-3} \mathrm{~min}^{-1}$ ) indicaram uma baixa estabilidade. $\mathrm{O}$ mesmo grau de estabilidade foi atribuído, respectivamente, aos $17 \%$ e $19 \%$ restantes dos cristais de hematita das amostras $\mathrm{H} 18$ e H22, dissolvidos no segundo seguimento linear, com valores médios de $k$ para as duas amostras de $2,35 \times 10^{-3} \mathrm{~min}^{-1}$. No restante das amostras hematíticas, o segundo segmento linear descreveu a dissolução de aproximadamente $30 \%$ dos cristais de hematita, classificados de média estabilidade, com valores de $k$ entre 1,5 e 1,9 x $10^{-3} \mathrm{~min}^{-1}$ (média de $1,67 \times 10^{-3} \mathrm{~min}^{-1}$ ). O segundo segmento linear nas amostras hematíticas refletiu, provavelmente, a dissolução da porção central dos cristais de hematita, cuja maior estabilidade pode estar relacionada ao aumento da concentração de alumínio nessa porção dos cristais, conforme indicado pela figura $1 \mathrm{~d}$. Este segmento pode ser conseqüência ainda da dissolução de pequenas quantidades de goethita (entre 3\% na H16 e 13\% na H13) presentes nas amostras hematíticas, conforme indicou a razão $\mathrm{Hm} /(\mathrm{Hm}+\mathrm{Gt})$ (Tabela 1$)$.

A dissolução de uma expressiva amplitude de cristais de goethita com média estabilidade (entre $10 \%$ na G3 e $64 \%$ na G2) nas amostras goethíticas latossólicas e plintossólica foi descrita, respectivamente, pelo segundo segmento linear das primeiras e o terceiro segmento da última, com valores médios de $k$ entre 0,4 e $1,5 \times 10^{-3} \mathrm{~min}^{-1}$ (média de $0,74 \mathrm{x}$ $10^{-3} \mathrm{~min}^{-1}$ ). Finalmente, o modelo descreveu ainda a dissolução de cristais de goethita com alta estabilidade, representada pelo terceiro segmento linear das amostras goethíticas latossólicas. Os valores de $k$ derivados destes segmentos variaram entre 0,03 e 0,10 x $10^{-3} \mathrm{~min}^{-1}$, com um valor médio de $0,075 \times 10^{-3} \mathrm{~min}^{-1}$. Um quinto grau de estabilidade, denominado de muito alta, foi atribuído aos cristais de goethita não solubilizados após os 3840 minutos de dissolução, representados pela diferença entre o teor inicial de ferro nas amostras e o teor de ferro dissolvido após a última etapa de dissolução (100 - \%Fe/SSL). A amplitude destes cristais variou entre $4 \%$ (G9) e 41\% (G11) nas amostras goethíticas latossólicas.

Considerando esta discriminação dos cristais, a qual permite comparar cristais de óxidos de ferro tanto entre amostras como dentro de uma mesma amostra, o modelo Avrami-Erofejev deve ser utilizado para descrever a dissolução de goethitas e hematitas em amostras de solos poligenéticos. Entretanto, tal comparação não pode ser conduzida a partir de parâmetros mineralógicos estimados pela metodologia tradicional, a qual estima parâmetros médios da população de cristais nas amostras, e sim por parâmetros estimados nos resíduos do material dissolvido ao longo das dissoluções (RUAN \& GILKES, 1995; INDA JUNIOR, 2002).

\section{CONCLUSÕES}

O modelo Kabai não discriminou diferentes fases de cristais de goethita e hematita nas amostras. A cinética dissolutiva de cristais de goethita e hematita derivada deste modelo permitiu somente a análise comparativa da estabilidade entre cristais de diferentes amostras. Neste caso, a substituição por $\mathrm{Al}^{3+}$ mostrou ser um parâmetro eficiente como indicativo da estabilidade à dissolução por redução para os cristais de goethita.

O modelo Avrami-Erofejev discriminou diferentes fases de goethita e hematita com estabilidades distintas nas amostras latossólicas e do Plintossolo. A cinética dissolutiva de cristais de goethita e hematita derivada deste modelo ofereceu condições para classificar o grau de estabilidade destes minerais dentro de cada amostra. A influência de parâmetros mineralógicos na cinética dissolutiva descrita por este modelo deve ser apoiada pela análise do resíduo do material dissolvido a cada etapa da dissolução.

Ambos os modelos indicaram que a hematita possui menor estabilidade à dissolução redutiva que a goethita, principalmente quando a última combina baixa substituição por $\mathrm{Al}^{3+}$ e alta ASE.

\section{REFERÊNCIAS BIBLIOGRÁFICAS}

CORNELL, R.M.; GIOVANOLI, R. Acid dissolution of hematites of different morphologies. Clay Minerals, v.28, p.223-232, 1993.

DICK, D.P. Caracterização de óxidos de ferro e adsorção de fósforo na fração argila de horizontes B latossólicos. 1986. 196f. Dissertação (Mestrado em Ciência do Solo) Curso de Pós-graduação em Ciência do Solo, Universidade Federal do Rio Grande do Sul. 
INDA JUNIOR, A.V. Caracterização de goethita e hematita em solos poligenéticos. 2002. 126f. Tese (Doutorado em Ciência do Solo) - Programa de Pós-graduação em Ciência do Solo, Universidade Federal do Rio Grande do Sul.

INDA JUNIOR, A.V.; KÄMPF, N. Avaliação de procedimentos de extração dos óxidos de ferro pedogênicos com ditionitocitrato-bicarbonato de sódio. Revista Brasileira de Ciência do Solo, v.27, p.1139-1147, 2003.

JEANROY, E. et al. Differential dissolution of hematite and goethite in dithionite and its implication on soil yellowing. Geoderma, v.50, p.79-94, 1991.

KABAI, J. Determination of specific activation energies of metal oxides and metal oxide hydrates by measurement of the rate of dissolution. Acta Chimica Academiae Scientiarum Hungaricae, v.78, p.57-73, 1973.

MEHRA, O.P.; JACKSON, M.L. Iron oxide removal from soils and clays by a dithionite-citrate system buffered with sodium bicarbonate. Proceedings Clays \& Clay Mineral Conference, v.7, p.317-327, 1960.

MUGGLER, C.C. Polygenetic oxisols on tertiary surfaces, Minas Gerais, Brazil. 1998. 186f. Tese (Doutorado em Ciência do Solo) - Wageningen Agricultural University.

RUAN, H.D.; GILKES, R.J. Acid dissolution of synthetic aluminous goethite before and after transformation to hematite by heating. Clay Minerals, v.30, p.55-65, 1995.

SCHULZE, D.G.; SCHWERTMANN, U. The influence of aluminium on iron oxides: X. Properties of Al-substituted goethites. Clay Minerals, v.19, p.521-539, 1984.

SCHULZE, D.G. The influence of aluminum on iron oxides: VIII. Unit-cell dimensions of Al-substituted goethites and estimation of Al from them. Clays and Clay Minerals, v.32, p.36-44, 1984
SCHWERTMANN, U.; KÄMPF, N. Properties of goethite and hematite in kaolinitic soils of southern and central Brazil. Soil Science, v.139, p.344-350, 1985.

SCHWERTMANN, U.; LATHAM, M. Properties of iron oxides in New Caledonian oxisols. Geoderma, v.39, p.105123, 1986.

SCHWERTMANN, U. Differenzierung der eisenoxide des bodens durch extraktion mit ammoniumoxalat-lösung. $\mathbf{Z}$ Pflanzenernähr Düng Bodenkd, v.105, p.194-202, 1964.

SCHWERTMANN, U. Solubility and dissolution of iron oxides. Plant and Soil, v.130, p.1-25, 1991.

SCHWERTMANN, U. The influence of aluminium on iron oxides: IX. Dissolution of Al-goethites in $6 \mathrm{M} \mathrm{HCl}$. Clay Minerals, v.19, p.9-19, 1984.

SCHWERTMANN, U. et al. The influence of aluminum on iron oxides. Part II. Preparation and properties of Al-substituted hematites. Clays and Clay Minerals, v.27, p.105-112, 1979.

SCHWERTMANN, U.; TAYLOR, R.M. Iron oxides. In: DIXON, J.B.; WEED, S.B. Minerals in soil environments. 2.ed. Madison: Soil Science Society of America, 1989. p.379-438.

SINGH, B.; GILKES, R.J. Properties and distribution of iron oxides and their association with minor elements in the soils of south-western Australia. Journal of Soil Science, v.43, p.77-98, 1992.

TORRENT, J. The reductive dissolution of synthetic goethite and hematite in dithionite. Clay Minerals, v.22, p.329337, 1987.

WELLS, M.A. et al. Properties and acid dissolution of metalsubstituted hematites. Clays and Clay Minerals, v.49, p.60-72, 2001. 